\title{
Construction of eukaryotic expression vector of TSLC1 gene
}

Qi-Lian Liang'1, Bi-Rong Wang ${ }^{2}$, Zhou-Yu Li', Guo-Qiang Chen'1 ${ }^{1}$ Yuan Zhou ${ }^{1}$

\begin{abstract}
1Department of Oncology, Affiliated Hospital of Guangdong Medical College, Zhanjiang, China

2Department of Chemotherapy, Affiliated Tumor Hospital of Guangzhou Medical College, Guangzhou, China
\end{abstract}

Submitted: 28 October 2010

Accepted: 4 December 2010

Arch Med Sci 2011; 7, 4: 579-585

DOI: 10.5114/aoms.2011.24124

Copyright @ 2011 Termedia \& Banach

\begin{abstract}
Introduction: To construct a eukaryotic expression vector of the tumour suppressor in lung cancer 1 (TSLC1) gene, so as to explore the mechanisms of tumour suppression of the gene theoretically.

Material and methods: The open reading frame (ORF) of TSLC1 gene was amplified with RT-PCR from normal human foreskin acrobystia, and cloned to pMD19-T simple vector (TA Clone method). The resultant plasmid was transformed into Escherichia coli JM109 for amplification. The TA Clone recombinant was digested by double restriction enzyme (BgI II/EcoR I) and analysed with agarose gel electrophoresis. The positive one was sequenced. The inserted DNA fragment was recovered, and then it was mounted into the eukaryotic expression vector pIRES2-EGFP, transformed into E. coli JM109 for amplification. A positive recombinant plasmid named pIRES2-EGFP-TSLC1 was confirmed by Bgl II/EcoR I double-enzyme digestion analysis.

Results: RT-PCR amplified the ORF of the TSLC1 gene. It was approximately 1400 base pairs. The obtained DNA was confirmed a high degree of homology with the sequence of TSLC1 CDNA sequence (AY358334) stored at GenBank.

Conclusions: Construction of a TSLC1 eukaryotic expression vector was successful, and it has established a solid foundation for further study.
\end{abstract}

Key words: TSLC1 gene, construction, eukaryotic expression vector.

\section{Introduction}

Cancer is still an inextirpable disease. However, human beings have evolved an array of host anti-tumour surveillance, to combat the arising malignant cell clones [1]. Tumour suppressor in lung cancer 1 (TSLC1) is a cloned tumour suppressor gene [2-5]. It is expressed in a variety of tissues and organs in the human body, particularly in the normal lung, brain, liver and skin tissues [6], but silenced in many cancers such as larynx cancer [7], cervical cancer [8], breast [9] and nasopharyngeal cancer [10]. It is widely considered to play a negative role in tumour genesis and development. Mao et al. [11] transfected recombinant adenovirus encoding TSLC1 cDNA into non-small cell lung cancer (NSCLC) A549 cell line, and found that $60 \%$ of cells underwent apoptosis as TSLC1 accumulated, and induced the activation of caspase 3 at the same. The suppressive role of TSLC1 in genesis and progression of tumours is not fully understood, but

\author{
Corresponding author: \\ Prof. Qi-Lian Liang \\ Department of Oncology \\ Affiliated Hospital \\ of Guangdong Medical College \\ 57 People Avenue \\ Zhanjiang 524001 \\ Guangdong, China \\ Phone: 86-759-2387455 \\ Fax: 86-759-2231754 \\ E-mail: lianqilian@gdmc.edu.cn
}


available data suggest that it regulates cell apoptosis and the cell cycle. So far, little is known about the effect of the TSLC1 gene on malignant cells; therefore, we constructed the TSLC1 gene eukaryotic expression vector, so as to establish a solid foundation for further study.

\section{Material and methods}

Normal foreskin acrobystia was obtained from patients admitted to Guangdong Medical College Hospital emergency operating room with written consent; TRIzol was purchased from Invitrogen (Carlsbad, CA, USA); Escherichia coli JM109 and plasmid pIRES2-EGFP were kind gifts from the biochemistry laboratory of Guangdong Medical College; High Fidelity PrimeScript ${ }^{\mathrm{TM}}$ RT-PCR Kit, DL2,000 DNA Marker, $\lambda$-Hind III DNA Marker, pMD19-T Simple vector, DNA A-Tailing Kit, DNA Ligation Kit, Agarose Gel DNA Purification Kit, restriction endonucleases EcoR I and $\mathrm{Bg}$ II were purchased from TaKaRa Biotechnology Co, Ltd (Dalian, China). Mini Plasmid Purification Kit was obtained from Beyotime Institute of Biotechnology (Haimen, China).

\section{Obtaining and identification of TSLC1}

\section{Design and synthesis of TSLC1 primer}

According to GenBank, accessible human TSLC1 gene mRNA sequence data (serial accession: AY 358334), using the primer design software Premier 5.0 to design primers containing Bgl II/EcoR I restriction site fragment for amplification of the ORF length of TSLC1. The primer was synthesized by TaKaRa Biotechnology Co, Ltd (Dalian, China) Primer: OUTER F 5'-GCGTGGGGTGCCCGACATGG-3' 20 mers

OUTER R 5'-AAATAGGGCCAGTTGGACAC-3' 20 mers.

\section{Total RNA extraction}

Up to $100 \mathrm{mg}$ of the normal foreskin (fat removed) specimen plus liquid nitrogen were ground to powder in a grinder followed by adding $1 \mathrm{ml}$ Trizol, and extraction of total RNA (according to the instructions of the Trizol kit).

\section{RT-PCR assay}

With the High Fidelity Prime Script ${ }^{\mathrm{TM}}$ RT-PCR Kit for (according to instructions of the kit) $P C R$ amplification, the $P C R$ conditions were: $94^{\circ} \mathrm{C}, 3 \mathrm{~min}$, 1 cycle; $98^{\circ} \mathrm{C}, 10 \mathrm{~s}, 55^{\circ} \mathrm{C}, 15 \mathrm{~s}, 72^{\circ} \mathrm{C}, 1 \mathrm{~min}, 30$ cycles; $72^{\circ} \mathrm{C}, 10 \mathrm{~min}, 1$ cycle.

\section{Recovery and purification of PCR product}

Take PCR products were loaded in $1 \times$ TAE buffer agarose gel electrophoresis, 120 V, $30 \mathrm{~min}$; agarose gel including the purpose of DNA was excised under the UV lamp, purified using TaKaRa Agarose Gel DNA Purification Kit according to the instructions, and TSLC1 fragments were recovered.

\section{TA Cloning}

One $\mu g$ of the recovered TSLC1 fragments was added " $A$ " tail with A-Tailing Kit; $5 \mu$ of the reaction solution was analysed with agarose gel electrophoresis; the new TSLC1 fragments were ligated to pMD19-T Simple vector with the proportion of $9: 1$ for $16^{\circ} \mathrm{C}, 30 \mathrm{~min} ; 10 \mu \mathrm{l}$ of pMD19-T Simple-TSLC1 solution was transformed into competent $E$. coli JM109 for amplification.

\section{Extraction and identification of pMD19-T simple-TSLC1 recombinant plasmid}

pMD19-T Simple-TSLC1 recombinant plasmid was extracted from E. coli JM109 with Mini Plasmid Purification Kit according to the instructions; $1 \mu \mathrm{g}$ of recombinant plasmid was digested by restriction enzyme Bgl II and EcoR I for $37^{\circ} \mathrm{C}, 8 \mathrm{~h} ; 5 \mu \mathrm{l}$ of digestion product was analysed with agarose gel electrophoresis; the positive plasmid was sequenced by TaKaRa Biotechnology Co, Ltd (Dalian, China).

\section{Subclone of pIRES2-EGFP-TSLC1 recombinant plasmid}

\section{Recovery of TSLC1}

Double restriction enzymes (Bgl II and EcoR I) digested pMD19-T Simple-TSLC1 recombinant plasmid, and the remaining steps were the same as "Recovery and purification of PCR product".

\section{Ligation of pIRES2-EGFP-TSLC1}

The recovered TSLC1 fragment was inserted to pIRES2-EGFP vector with the concentration proportion of $9: 1$ for $16^{\circ} \mathrm{C}, 8 \mathrm{~h}$ (according to DNA Ligation Kit instructions), and then transformed into competent E. coli JM 109 for amplification.

\section{Extraction and identification of pIRES2-EGFP- TSLC1 recombinant plasmid}

This step is the same as "Extraction and identification of PMD19-T simple-TSLC1 recombinant" except for DNA sequencing.

\section{Results}

\section{Evaluation of RT-PCR product}

The total RNA extracted from normal foreskin tissue was reversely transcribed to CDNA as a template for PCR, and the full length of $1400 \mathrm{bp}$ of TSLC1 was obtained. Figure 1 depicts the result 
of purification of the TSLC1 gene fragment after adding the "A" tail. Figures 2 and 3 show the basic structure and restriction sites of plasmid pMD19-T Simple and pIRES2-EGFP respectively.

\section{Evaluation of TA Clone recombinant plasmid}

pMD19-T Simple-TSLC1 recombinant plasmid was digested by restriction enzymes BgI II and EcoR I and analysed with agarose gel electrophoresis. The positive plasmid was sequenced. The result is shown in Figure 4. Obtained data were aligned with the sequences deposited in GenBank (AY 358334).

\section{Evaluation of pIRES2-EGFP-TSLC1 recombinant plasmid}

pIRES2-EGFP-TSLC1 recombinant plasmid was digested by restriction enzymes BgI II and EcoR I, and then analysed together with pIRES2-EGFP vector (after being digested by restriction enzymes BgI II and EcoR I) and TSLC1 (from "Recovery of TSLC1") recovered with agarose gel electrophoresis (Figure 5). The result confirmed that the pIRES2EGFP-TSLC1 eukaryotic expression vector was successfully constructed.

\section{Discussion}

Since identification of TSLC1, many scholars have found that it was low or missing expression in a variety of human cancer tissues, while widely present in normal human tissues, especially in the skin, lungs and liver tissues. Therefore, TSLC1 is widely considered as a tumour suppressor gene. The gene has been a hot spot in cancer research, especially the mechanisms of potential to induce apoptosis in malignant cells. In this study, extracts from skin and constructs TSLC1 gene eukaryotic
M

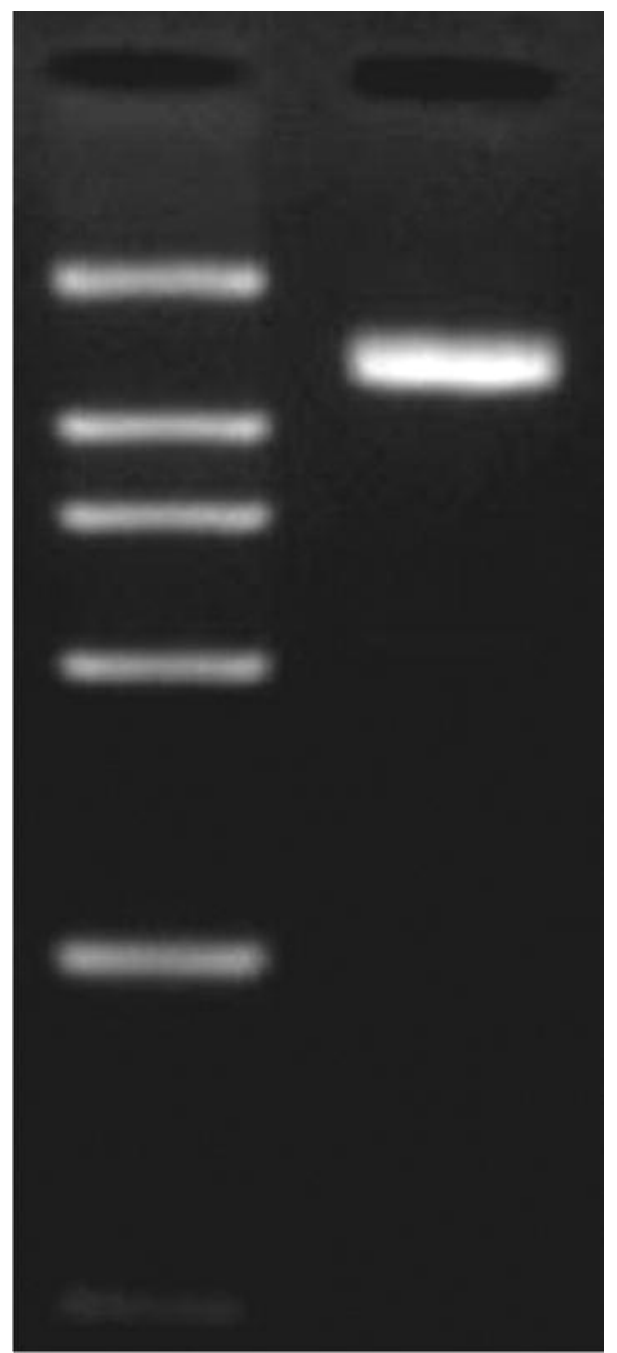

Figure 1. Purification of the TSLC1 gene fragment M - DL2.000 DNA marker, 1 - TSLC1-PCR product (added "A" tail)

BcaBEST ${ }^{\mathrm{TM}}$ sequencing primer RV-M

GAGCGGATAACAATTTCACACAGG $\longrightarrow$ ECOR V

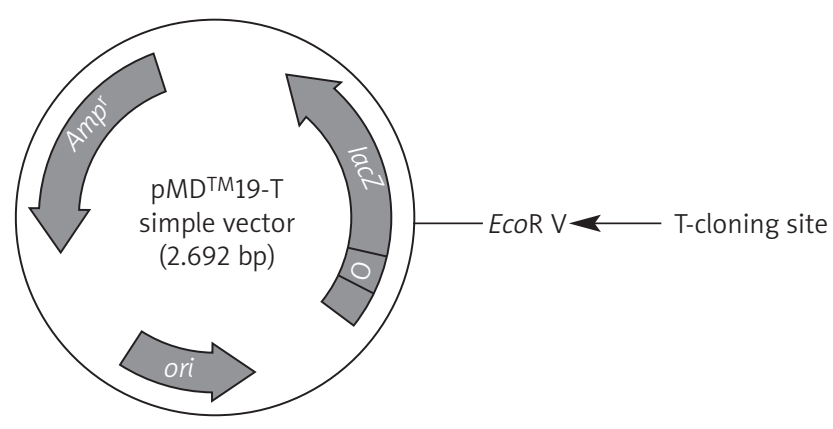

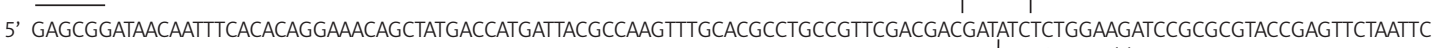
ACTGGCCGTCGTTTTACAACGTCGTGACTGGGAAAACCCTGGCG 3' $\leftarrow$ CAGCACTGACCCTTTGGGACCGC BCeBEST ${ }^{\mathrm{TM}}$ sequencing primer M13-47

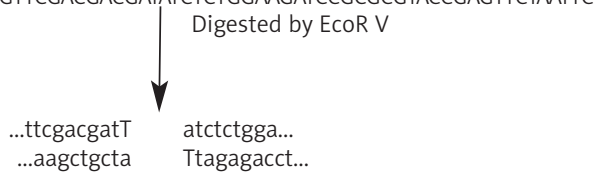

Figure 2. The structure and important restriction sites of pMD19-T simple vector 
pIRES2-EGFP vector information

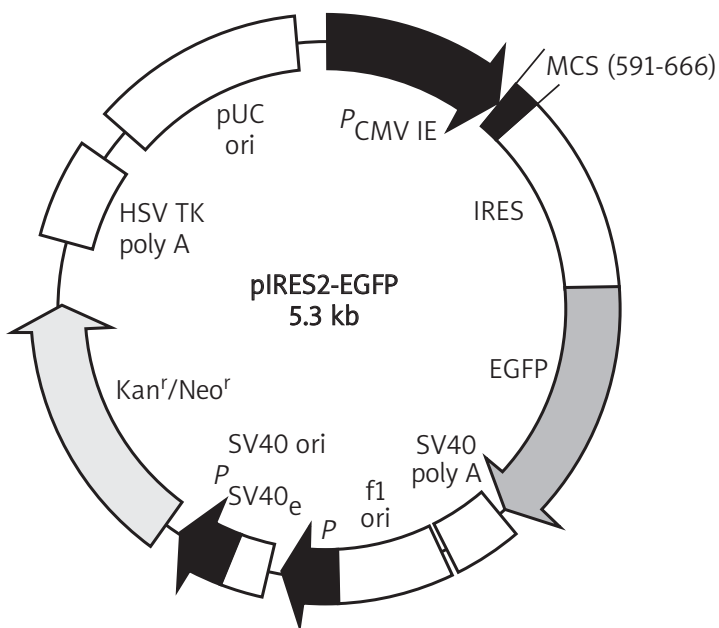

$\begin{array}{llllllll}591 & 601 & 611 & 621 & 631 & 641 & 651\end{array}$

GCTAGCGCTACCGGACTCAGATCTCGAGCTCAAGCTTCGAATTCTGCAGTCGACGGTACCGCGGGCCCGGGATCC... IRES

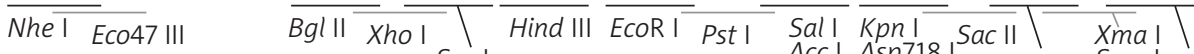

Apa I Sma I BamH I Bsp120 I

Figure 3. The structure and digestion sites of pIRES2-EGFP plasmid
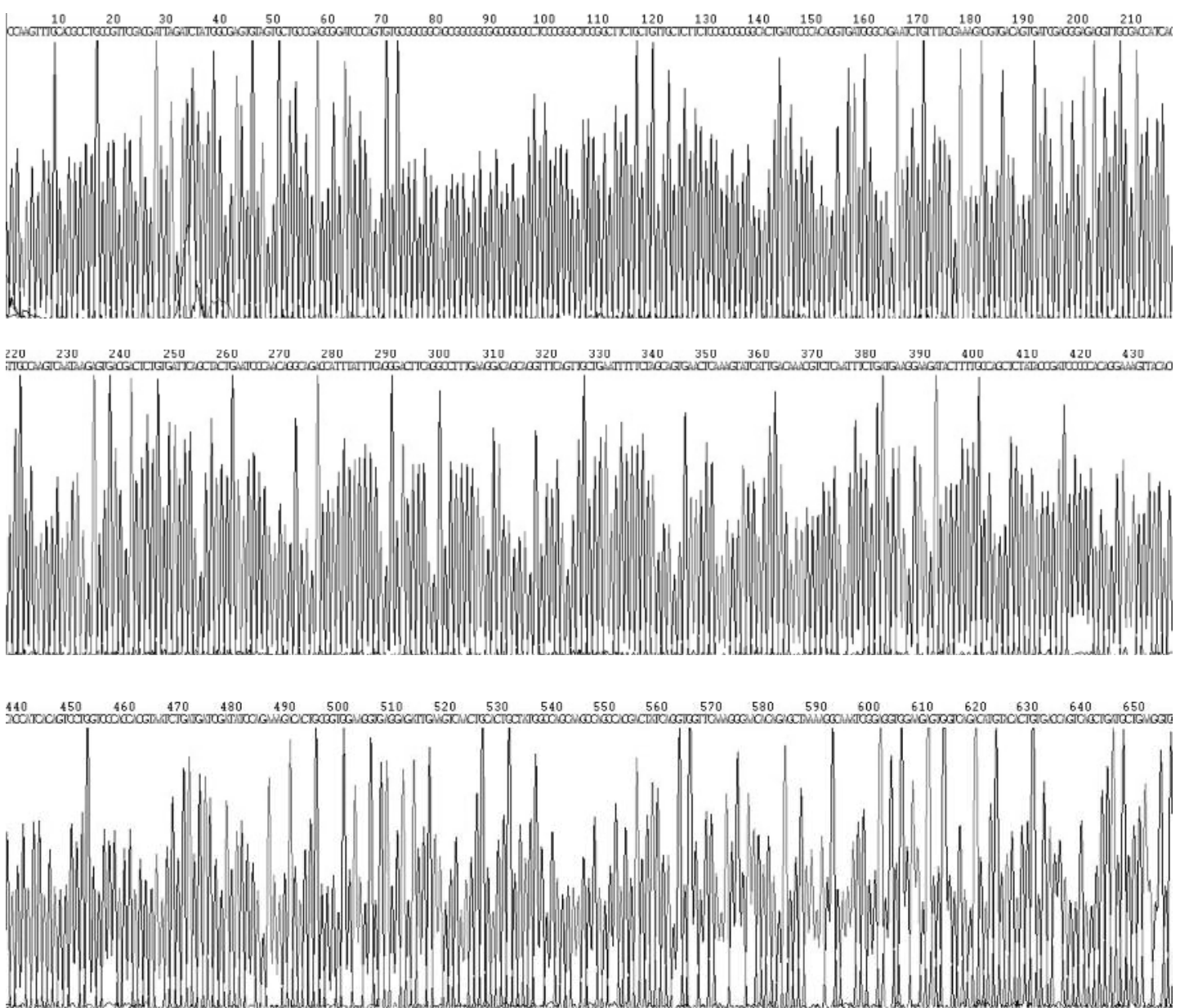

Figure 4. Full-length 1393 bp of the TSLC1 gene sequence diagram 

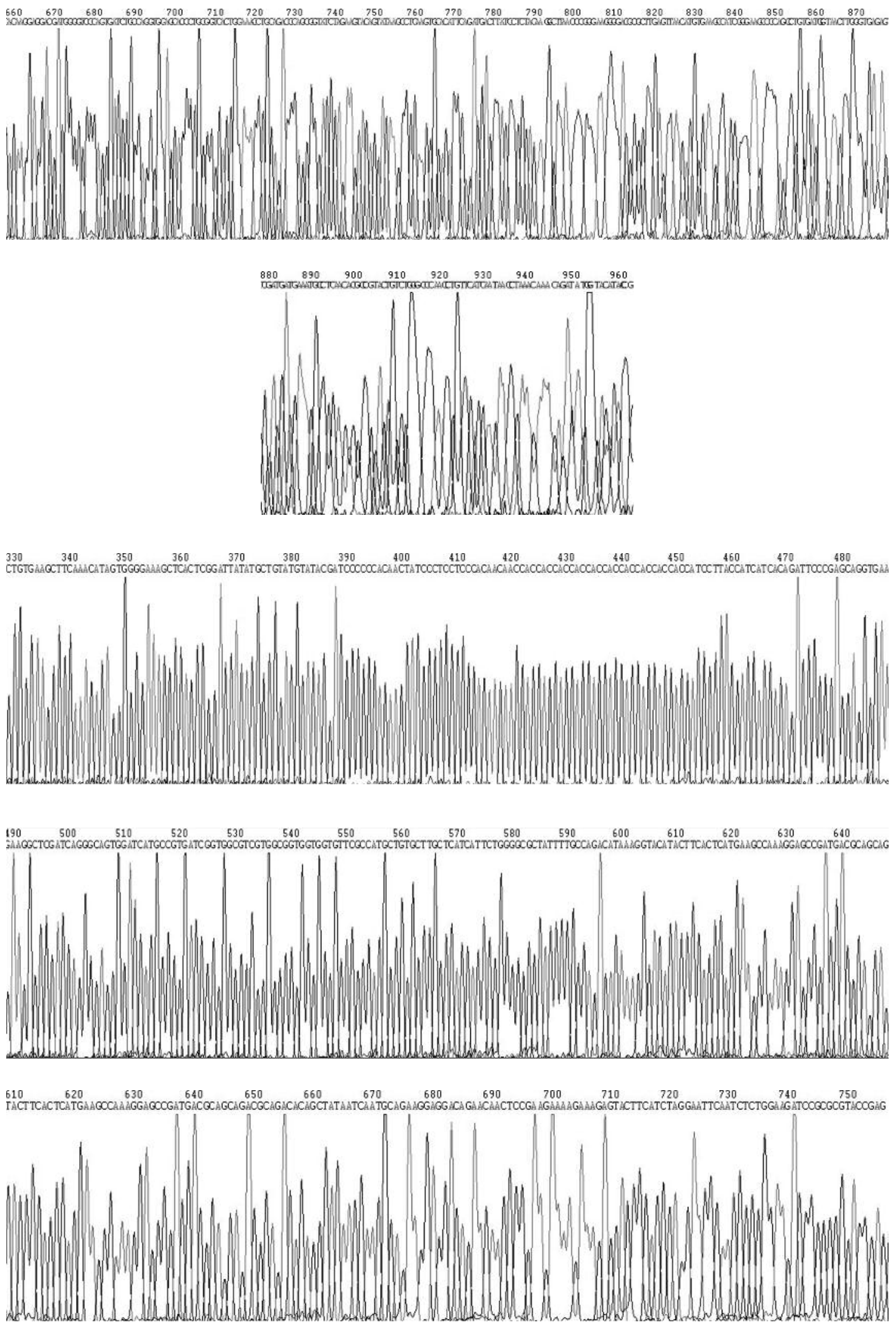

Figure 4. Cont 


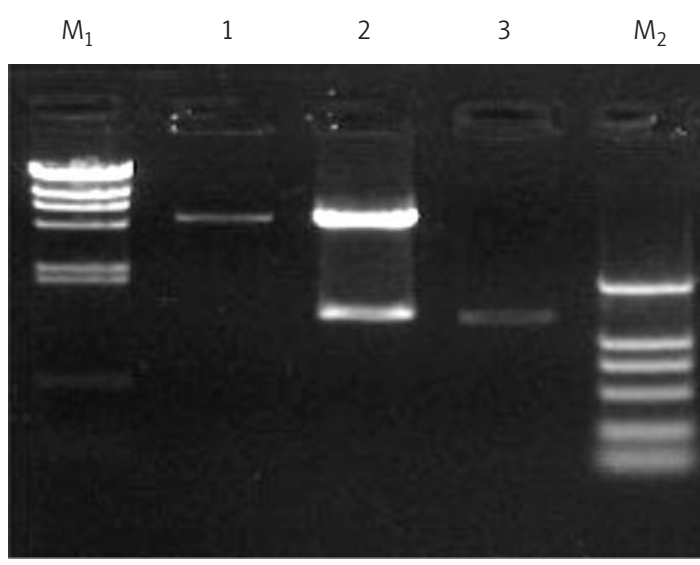

Figure 5. Bgl II/EcoR I double-enzyme digestion analysis of pIRES2-EGFP-TSLC1 recombinant plasmid $M_{1}-\lambda$-Hind III DNA marker $\lambda, 1$ - pIRES2-EGFP vector, 2 - pIRES2-EGFP-TSLC1 double-enzyme digestion, 3 - TSLC1 INSERT, M2 - DL2,000 DNA marker

expression vector for further study of the mechanism of tumour suppressor genes.

The key of the TSLC1 gene clone is the vector; it determines whether the exogenous gene can express steadily after being transfected into cells. The present study shows that the pIRES2-EGFP eukaryotic expression vector has the following advantages: 1) It contains internal ribosome entry site 2 (IRES2) and enhanced green fluorescent protein (EGFP) clone regions, enable transiently transfected into mammalian cells to express EGFP with high selectivity; furthermore, it is able not only to express EGFP alone but also to obtain a stable transfected cell line. 2) The EGFP is a kind of wildtype GFP, with characteristics of highly selective fluorescence and highly expressed in mammalian cells. Two amino acids (Phe-64 and Ser-65) in GFP mut1 variant6 of EGFP can be replaced by Leu and Thr, which makes its green fluorescence intensity increase greatly, thus screening exogenous gene expression in mammalian cells easily. 3) It contains an immediate early strong promoter from cytomegalovirus, which is conducive to a high level of transcription; furthermore, the EGFP gene downstream contains SV40 polyA signal, which can improve the stability of RNA. 4) It contains the neomycin and kanamycin resistance gene and the HSV TK gene poly A signal, which are favourable to screening positive cells after cell transformation or transfection [12-14].

Designing a pair of primers with Bgl II/EcoR I restriction enzyme sites to amplify the ORF of TSLC1, and mounted to PMD19-T Simple vector, E. coli transformation, sequencing, etc., have confirmed that the obtained cDNA extracted from the skin is submitted to GenBank release (accession number: AY 358334). In the current study, this DNA Ligation Kit is different from the ordinary Taq ligase enzyme, which can ligate the TSLC1 fragment into the pIRES2-EGFP eukaryotic expression vector exactly and efficiently after double restriction enzyme digestion, without needing to consider the pros and cons ends of ligating fragments. The recombinant plasmid was excised out by Bgl II/ EcoR I double-restriction enzyme digestion and analysed by agarose gel electrophoresis. The result shows that the ligated fragments were about $1.4 \mathrm{~kb}$ and $5.3 \mathrm{~kb}$, which reveals that the TSLC1 gene and pIRES2-EGFP vector size was corrected, and the TSLC1 eukaryotic expression vector has been constructed successfully.

\section{Acknowledgments}

Project supported by Science and Technology Planning Project of Guangdong Province, China (No. 2007B031513003).

\section{References}

1. Gao Y, Xu X, Dong Z, et al. A survey on the distribution of healthy people with different anti-tumour ability. Arch Med Sci 2010; 6: 806-14.

2. Dickinson PJ, Surace El, Cambell M, et al. Expression of the tumor suppressor genes NF2, 4.1B, and TSLC1 in canine meningiomas. Vet Pathol 2009; 46: 884-92.

3. Kitamura Y, Kurosawa G, Tanaka M, et al. Frequent overexpression of CADM1/IGSF4 in lung adenocarcinoma. Biochem Biophys Res Commun 2009; 12: 480-4.

4. Ohno N, Terada N, Komada M, et al. Dispensable role of protein 4.1B/DAL-1 in rodent adrenal medulla regarding generation of pheochromocytoma and plasma lemmal localization of TSLC1. Biochim Biophys Acta 2009; 1793: 506-15.

5. Hurtubise A, Bernstein ML, Momparler RL. Preclinical evaluation of the antineoplastic action of 5-aza-2'deoxycytidine and different histone deacetylase inhibitors on human Ewing's sarcoma cells. Cancer Cell Int 2008; 8: 16.

6. Shingai T, Ikeda W, Kakunaga S, et al. Implications of nectin-like molecule-2/IGSF4/RA175/SgIGSF/TSLC1/ SynCAM1 in cell-cell adhesion and transmembrane protein localization in epithelial cells. J Biol Chem 2003; 278: 35421-7.

7. Wang JL, Zhang HZ, Bai WS, Liu L, Bian K, Cheng SY. Promoter hypermethylation and mRNA expression of TSLC1 gene in laryngeal squamous cell carcinoma [Chinease]. J Fourth Mil Med Univ 2007; 28: 651-3.

8. Yang YX, Yang AH, Yang ZJ, Wang ZR, Xia XH. Involvement of tumor suppressor in lung cancer 1 gene expression in cervical carcinogenesis. Int J Gynecol Cancer 2006; 16: 1868-72.

9. Heller G, Geradts J, Ziegler B, et al. Downregulation of TSLC1 and DAL-1 expression occurs frequently in breast cancer. Breast Cancer Res Treat 2007; 103: 283-91.

10. Lung HL, Cheung AK, Xie D, et al. TSLC1 is a tumor suppressor gene associated with metastasis in nasopharyngeal carcinoma. Cancer Res 2006; 66: 9385-92.

11. Mao X, Sendlitz E, Truant R, et al. Re-expression of TSLC1 in a non-small-cell lung cancer cell line induces apoptosis and inhibits tumor growth. Oncogene 2004; 23: 5632-42.

12. Marx JC, Allay JA, Persons DA, et al. High-efficiency transduction and long-term gene expression with 
a murine stem cell retroviral vector encoding the green fluorescent protein in human marrow stromal cells. Hum Gene Ther 1999; 10: 1163-73.

13. Persons DA, Allay JA, Allay ER, et al. Retroviral-mediated transfer of the green fluorescent protein gene into murine hematopoietic cells facilitates scoring and selection of transduced progenitors in vitro and identification of genetically modified cells in vivo. Blood 1997; 90: 1777-86.

14. Wang X, Wan H, Korzh V, Gong Z. Use of an IRES bicistronic construct to trace expression of exogenously introduced mRNA in zebrafish embryos. Biotechniques 2000; 29: 814-6, 818, 820 . 